\title{
BMJ Open Bone mineral density and fracture risk with long-term use of inhaled corticosteroids in patients with asthma: systematic review and meta-analysis
}

\author{
Yoon K Loke, Daniel Gilbert, Menaka Thavarajah, Patricia Blanco, \\ Andrew M Wilson
}

To cite: Loke YK, Gilbert D, Thavarajah $\mathrm{M}$, et al. Bone mineral density and fracture risk with long-term use of inhaled corticosteroids in patients with asthma: systematic review and metaanalysis. BMJ Open 2015;5: e008554. doi:10.1136/ bmjopen-2015-008554

- Prepublication history and additional material is available. To view please visit the journal (http://dx.doi.org/ 10.1136/bmjopen-2015008554).

Received 23 April 2015 Revised 22 October 2015 Accepted 23 October 2015

CrossMark

Norwich Medical School, University of East Anglia, Norwich, UK

Correspondence to Professor Yoon K Loke; y.loke@uea.ac.uk

\section{ABSTRACT}

Objectives: We aimed to assess the association between long-term use of inhaled corticosteroids (ICS) and bone adverse effects in patients with asthma. Design: Systematic review and meta-analysis of fracture risk and changes in bone mineral density with long-term ICS use in asthma.

Methods: We initially searched MEDLINE and EMBASE in July 2013, and performed an updated PubMed search in December 2014. We selected randomised controlled trials (RCTs) and controlled observational studies of any ICS (duration at least 12 months) compared to non-ICS use in patients with asthma. We conducted meta-analysis of ORs for fractures, and mean differences in bone mineral density. Heterogeneity was assessed using the $\mathrm{I}^{2}$ statistic.

Results: We included 18 studies (7 RCTs and 11 observational studies) in the systematic review. Metaanalysis of observational studies did not demonstrate any significant association between ICS and fractures in children (pooled OR $1.02,95 \% \mathrm{Cl} 0.94$ to 1.10 , two studies), or adults (pooled OR $1.09,95 \% \mathrm{Cl} 0.45$ to 2.62 , four studies). Three RCTs and three observational studies in children reported on bone mineral density at the lumbar spine, and our meta-analysis did not show significant reductions with ICS use. Three RCTs and four observational studies in adults reported on ICS use and bone mineral density at the lumbar spine and femur, with no significant reductions found in the meta-analysis compared to control.

Conclusions: ICS use for $\geq 12$ months in adults or children with asthma was not significantly associated with harmful effects on fractures or bone mineral density.

\section{INTRODUCTION}

Asthma is a chronic inflammatory condition that affects both adults and children. There is a substantial body of evidence that suggest inhaled corticosteroids (ICS) are effective at controlling symptoms, improving lung

\section{Strengths and limitations of this study}

- Comprehensive search of two databases with independent study selection and data extraction.

- Included both observational and randomised studies in adults and/or children with asthma.

- Heterogenous nature of studies and the outcome measures which were available for analysis.

- Inability to properly assess differences between drugs, type of inhaler device or doseresponsiveness.

function and reducing acute exacerbations. ${ }^{1}$ They are therefore considered the gold standard first-line preventative therapy and are widely recommended in national and international guidelines. ${ }^{2} 3$

However, long-term ICS use may be associated with adverse effects such as cataract, osteoporosis, fractures and reduction in growth velocity in children. ${ }^{4}$ Concerns surrounding these potential harms may have a negative effect on ICS adherence, thus exposing patients to poorer asthma control and a potentially higher risk of needing oral corticosteroids for acute exacerbations. ${ }^{4}$ Certain age groups, such as children or postmenopausal women may be particularly susceptible to adverse effects on bone metabolism and formation, and this therefore remains an area of concern for these patients.

The existing meta-analyses of ICS and bone adverse effects have usually included data from participants with chronic obstructive pulmonary disease (COPD) ${ }^{5-7}$ and to date, there has been less focus on the effects in asthma alone. Patients with asthma may not share the same susceptibilities to osteoporosis as the patient with COPD because of differences in risk factors such as cigarette consumption, multimorbidity and nutritional problems that are prevalent in patients with COPD. ${ }^{8}{ }^{9}$ It 
therefore remains unclear whether patients with asthma have a greater or lesser risk of bone adverse effects than those with COPD and a further review is necessary to clarify these risks for patients with asthma alone.

Hence we aimed to analyse the effects of long-term $(\geq 12$ months) ICS use in patients with asthma alone, concentrating on fracture and bone mineral density (BMD) outcomes.

\section{METHODS}

\section{Study selection criteria}

We aimed to focus on long-term, important but infrequent adverse effects on bone and as such, eligible studies had to have $>20$ users of each ICS formulation, with follow-up of at least 12 months in duration.

Our inclusion criteria for RCTs were (1) parallel-group RCT; (2) participants with asthma of any severity; (3) ICS as the intervention versus a control treatment, where the comparison groups consisted of ICS versus other asthma therapy (or placebo), or ICS in combination with longacting $\beta$-agonist (LABA) versus a LABA alone; and (4) stated aim to evaluate fractures or BMD.

We also evaluated controlled observational studies (case control, prospective cohort or retrospective cohort) reporting on risk of fractures or change in BMD with any ICS exposure compared to those without ICS exposure.

\section{Exclusion criteria}

We excluded studies that recruited mixed groups of participants (asthma/COPD) if the outcomes were not separately reported according to specific disease condition. We excluded crossover trials and studies that considered only oral corticosteroid use without reporting the effects of inhaled corticosteroids.

\section{Search strategy}

We initially searched MEDLINE and EMBASE in June 2013 using a broad strategy for a wide range of adverse effects potentially associated with ICS use, and we subsequently updated this through a more focused PubMed search in December 2014 (see eAppendix 1 for search terms and restrictions). We also manually looked through the bibliographies of included studies as well as existing systematic reviews for any other articles that may be potentially suitable.

\section{Study selection}

Two reviewers (MT and PB) independently, and in duplicate scanned all titles and abstracts and excluded articles that clearly were not RCTs or observational studies of ICS in patients with asthma. We proceeded to assess full-text versions of potentially relevant articles and conducted more detailed checks against our eligibility criteria, focusing on bone and fracture adverse effects. A third researcher (YKL or AMW) evaluated the decision on inclusion or exclusion in discussion with the two reviewers.

\section{Study characteristics and data extraction}

We used preformatted tables to record study design and participant characteristics, definition of asthma, pharmacological agent (dose, device and frequency), and duration of follow-up. Two reviewers independently extracted data (MT and PB) on relevant outcomes, where we prespecified fracture risk of primary interest, and BMD at the lumbar spine or the femur as secondary end points. Any discrepancies were resolved through the involvement of a third reviewer (DG or YKL or AMW) after rechecking the source papers.

\section{Risk of bias assessment}

Two reviewers independently assessed the reporting of blinding of participants and personnel, randomisation sequence, allocation concealment, withdrawals and the loss to follow-up in RCTs. In order to assess validity of the associations between adverse effects and ICS use, we extracted information on participant selection, ascertainment of exposure and outcomes, and methods of addressing confounding in observational studies. ${ }^{10}$

We aimed to use a funnel plot and asymmetry testing to assess publication bias provided that there were more than 10 studies in the meta-analysis, and the absence of significant heterogeneity. ${ }^{11}$

\section{Statistical analysis}

We pooled trial data using Review Manager (RevMan) V.5.3.2 (Nordic Cochrane Center, Copenhagen, Denmark). We used the inverse variance method to pool ORs for fracture events, and mean differences for $\operatorname{BMD}\left(\mathrm{g} / \mathrm{cm}^{2}\right)$. In accordance with the recommendations of the Cochrane Handbook, we derived any SDs from $95 \%$ CIs or $p$ values. ${ }^{12}$ We assessed statistical heterogeneity using the $\mathrm{I}^{2}$ statistic with $\mathrm{I}^{2}>50 \%$ indicating a substantial level of heterogeneity.

If a trial had more than one group of non-ICS users as controls, we analysed data for ICS versus placebo (if available) in preference to data from active comparators such as ICS versus nedocromil, montelukast or disodium cromoglycate. If combination formulations were evaluated in the trial, we chose unconfounded comparisons based on ICS used together with the other drug versus other drug alone.

If a trial had several arms involving different ICS doses, we combined all the ICS arms together as recommended by the Cochrane Handbook. ${ }^{13}$

We did not have a pre-registered protocol.

\section{RESULTS}

We screened 1887 potentially relevant articles, and finally included 18 studies in our systematic review (comprising 7 RCTs, ${ }^{14-20}$ and 11 observational studies). ${ }^{21-31}$ The process of study selection is shown in figure 1 .

Table 1A, B show the characteristics of the included RCTs, and the observational studies, respectively. 
Tables 2 and 3 report on study validity and outcomes in adult and children, respectively.

Four of the RCTs focused solely on children, ${ }^{14} 151920$ while the remaining three were in adults. ${ }^{16-18}$ Treatment duration was up to 4 years in one study, ${ }^{15}$ while the remaining six trials had ICS therapy for between 52 and 104 weeks. Intervention arms of the trials included fluticasone ( 5 trials), budesonide (3 trials) and mometasone (one trial). Fluticasone and mometasone were the ICS used in the intervention arms of one trial, and in this trial, we evaluated the results of all ICS users combined against montelukast. ${ }^{18}$

Five of the observational studies focused solely on children, ${ }^{21-23} 2529$ while the remainder looked at adults or a mixture of age groups. The observational studies looked at wider range of ICS than the RCTs, with the inclusion of beclometasone, flunisolide and triamcinolone users.

\section{Study validity}

Validity assessment of the included studies is reported in tables 2 and 3.

\section{Randomised controlled trials $(\mathrm{n}=7)$}

Overall, four of the RCTs reported an appropriate method of sequence generation, while five provided details on how concealment of allocation was achieved. With regards to blinding, five trials reported the use of double-blinding. Ascertainment of BMD was consistently performed through dual-energy X-ray absorptiometry
(DEXA) scans, but the trials did not state how and when fracture diagnoses were confirmed. One major limitation that affected all the trials stemmed from discontinuations and substantial losses to follow-up for measurement of BMD outcomes at final time-points.

\section{Observational studies $(n=11)$}

We felt that only four studies took account of a good range of variables when tackling baseline confounding. ${ }^{26} 2729{ }^{30}$ Assessment of compliance or adherence to ICS use was reported in four studies. ${ }^{21} 2230{ }^{31}$ Fracture events were typically recorded through administrative codes while one study relied on patient self-report. Ascertainment of BMD was through DEXA scans. Overall, we felt that most of the studies were at moderate to high risk of bias due to the above limitations, with four studies possibly of slightly better methodological quality because of adequate outcome ascertainment and adjustment for confounders. ${ }^{26} 272930$

\section{Fractures with ICS}

We identified one large long-term RCT in children that reported adjusted fracture rate of 5.7 per 100 patient years with budesonide as compared to 5.1 per 100 patient years with placebo $(p=0.53) .{ }^{32}$ Similarly, there was no significant increase in likelihood of fracture in a meta-analysis of two observational studies in children, (OR 1.02, 95\% CI 0.94 to $\left.1.10, \mathrm{I}^{2}=0 \%\right)^{27} 29$ as shown in figure 2. The point estimates of fracture risk was not
Figure 1 Flow diagram of study selection. BMD, bone mineral density; RCT, randomised controlled trial.

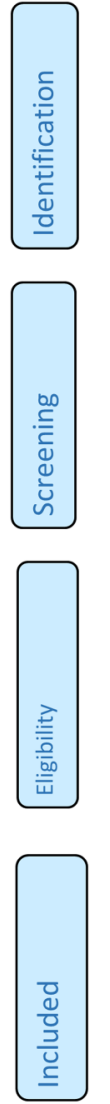




\section{(A) Randomised controlled trials}

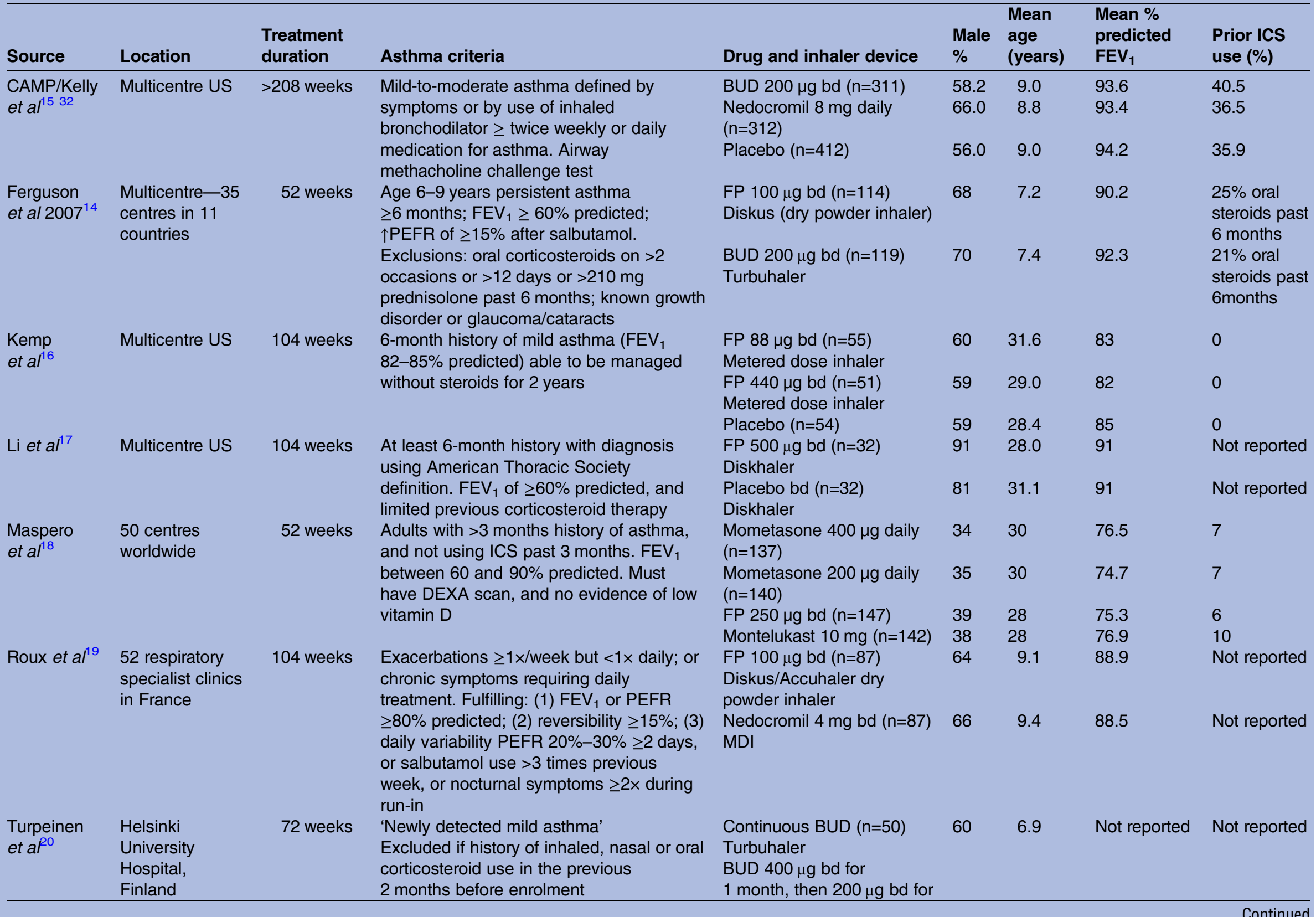


Table 1 Continued

(A) Randomised controlled trials

\begin{tabular}{|c|c|c|c|c|c|c|c|c|}
\hline Source & Location & $\begin{array}{l}\text { Treatment } \\
\text { duration }\end{array}$ & Asthma criteria & Drug and inhaler device & $\begin{array}{l}\text { Male } \\
\%\end{array}$ & $\begin{array}{l}\text { Mean } \\
\text { age } \\
\text { (years) }\end{array}$ & $\begin{array}{l}\text { Mean \% } \\
\text { predicted } \\
\text { FEV }_{1}\end{array}$ & $\begin{array}{l}\text { Prior ICS } \\
\text { use (\%) }\end{array}$ \\
\hline
\end{tabular}

2nd-6th months, then

$100 \mu \mathrm{g}$ bd for final

12 months.

BUD/placebo $(n=44) \quad 66 \quad 6.7 \quad$ Not reported Not reported

Turbuhaler

BUD $400 \mu \mathrm{g}$ bd for $1 \mathrm{st}$

month, then $200 \mu \mathrm{g}$ bd for

2nd to 6th months, then

placebo for final 12 months

Sodium cromoglicate-

$10 \mathrm{mg}$ tds for 18 months

50

7.0

Not reported Not reported

MDI

Bd, two times a day; BUD, budesonide; DEXA, dual-energy X-ray absorptiometry; FEV ${ }_{1}$, forced expiratory volume in 1 s; FP, fluticasone propionate; ICS, inhaled corticosteroids; MDI, metered dose inhaler; PEFR, peak expiratory flow rate.

\begin{tabular}{|c|c|c|c|c|c|}
\hline \multicolumn{6}{|c|}{ (B) Observational studies } \\
\hline Study & Design & $\begin{array}{l}\text { Adverse } \\
\text { effects } \\
\text { measured }\end{array}$ & $\begin{array}{l}\text { Data source and number of } \\
\text { patients }\end{array}$ & $\begin{array}{l}\text { Selection of patients: asthma definition } \\
\text { and patient characteristics (or selection of } \\
\text { cases and controls) }\end{array}$ & Type of ICS \\
\hline $\begin{array}{l}\text { Agertoft and } \\
\text { Pedersen }\end{array}$ & Cross-sectional study & BMD & $\begin{array}{l}\text { Outpatient paediatric clinic, Kolding } \\
\text { Hospital, Denmark } \\
157 \text { cases, } 111 \text { controls }\end{array}$ & $\begin{array}{l}\text { Selection of cases: Children with persistent } \\
\text { asthma and no other chronic disease, on ICS } \\
\text { continuously for } \geq 3 \text { years. } \\
\text { Mean age: } 10.3 \text { years, male } 69 \%, \% F E V_{1} \\
\text { predicted: } 97 \\
\text { Selection of controls: asthmatic children, who } \\
\text { have never taken inhaled/systemic } \\
\text { corticosteroids for }>2 \text { weeks per year. } \\
\text { Mean age: } 9.9 \text { years, male } 55 \%, \% F E V_{1} \\
\text { predicted: } 81\end{array}$ & BUD \\
\hline Allen et $a P^{2}$ & Prospective & BMD & $\begin{array}{l}\text { Department of Paediatrics, Royal } \\
\text { North Shore Hospital, Sydney, } \\
\text { Australia } \\
48 \text { cases, } 9 \text { controls }\end{array}$ & $\begin{array}{l}\text { Selection of cases: prepubertal asthmatic } \\
\text { children requiring }>3 \text { courses oral } \\
\text { corticosteroids within study period } \\
\text { Mean age: } 7.8 \text { years, male } 63 \% \\
\text { Selection of controls: children not using } \\
\text { corticosteroids. } \\
\text { Mean age: } 8.4 \text { years, male } 78 \%\end{array}$ & BDP, BUD \\
\hline
\end{tabular}




\section{(B) Observational studies}

\begin{tabular}{llll} 
Study & Design & $\begin{array}{l}\text { Adverse } \\
\text { effects } \\
\text { measured }\end{array}$ & $\begin{array}{l}\text { Data source and number of } \\
\text { patients }\end{array}$ \\
\hline $\begin{array}{l}\text { Bahceciler } \\
\text { et } a \text { f }^{3}\end{array}$ & Cross-sectional study & BMD & $\begin{array}{l}\text { Outpatient Allergy Clinic of Marmara } \\
\text { University Hospital, Istanbul, Turkey } \\
52 \text { cases, 22 controls }\end{array}$
\end{tabular}

El et $a$ R $^{4} \quad$ Observational BMD

Harris et $a^{25} \quad$ Cross-sectional study

Johannes et $a^{\text {R6 }}$

\section{Nested case-control study Risk of \\ non-vertebral} fracture
Ingenix Epidemiology-Research database of United Healthcare members, 17 states in the USA. 1722 cases, 17220 controls
Selection of patients: asthma definition and patient characteristics (or selection of cases and controls)

Asthma definition: mild intermittent plus

BUD persistent mild to moderate asthma

Selection of cases: Children treated for $\geq 6$ months.

Mean age: 6.4 years, male $42 \%$

Characteristics of high-dose ICS group: Mean age: 3 years

Mean duration of disease: 50.4 months

Characteristics of low dose ICS group:

Mean age: 5.8 years

Mean duration of disease: $\mathbf{3 8 . 3}$ months

Selection of controls: Age-matched asthmatic children who have never received ICS

Mean age: 6.8 years, male $45 \%$

Outpatients, Dokuz Eylul University Balcova, Izmir, Turkey

Asthma severity defined according to Global Initiative for Asthma guideline

Selection of cases: patients with mild or moderate asthma and regular ICS use. Mean age: 44.04 years, male $0 \%, \% F$ : 89.71

Controls : Mean age: 44.43 years, male $0 \%$ Selection of subjects:

Prepubertal asthmatic children stratified into groups according to corticosteroid treatment received in the past 6 months

1) no inhaled corticosteroid, mean age:

8.2 years, male $70 \%$

2) moderate dose inhaled corticosteroid (400$800 \mu \mathrm{g} /$ day), mean age: 7.4 years, male $56 \%$ 3) high-dose inhaled corticosteroid (>800 $\mu \mathrm{g} /$ day), mean age: 8.9 years, male $75 \%$,

Adults $\geq 40$ years age, in health plan for $\geq 12$ continuous months Jan 1997 to Jun 2001 , with ICD-9 code for asthma, or COPD

BDP, BUD, FP flunisolone, triamcinolone

Selection of cases: Non-vertebral fractures by ICD-9 codes, with claim for treatment (including inpatient hip fractures) Mean age 52.9 years, male $29.4 \%$
Not specified

BDP, BUD, FP 
Table 1 Continued

(B) Observational studies

\begin{tabular}{|c|c|c|c|c|c|}
\hline Study & Design & $\begin{array}{l}\text { Adverse } \\
\text { effects } \\
\text { measured }\end{array}$ & $\begin{array}{l}\text { Data source and number of } \\
\text { patients }\end{array}$ & $\begin{array}{l}\text { Selection of patients: asthma definition } \\
\text { and patient characteristics (or selection of } \\
\text { cases and controls) }\end{array}$ & Type of ICS \\
\hline & & & & $\begin{array}{l}\text { Selection of controls: } \\
\text { Sampled from person-time of respiratory } \\
\text { cohort by two-tiered random sampling with } \\
\text { replacement } \\
\text { Mean age } 52.2 \text { years, male } 41.1 \%\end{array}$ & \\
\hline $\begin{array}{l}\text { Schlienger } \\
\text { et } a f^{7}\end{array}$ & $\begin{array}{l}\text { Retrospective } \\
\text { Population-based nested } \\
\text { case-control analysis }\end{array}$ & Fracture risk & $\begin{array}{l}\text { UK General Practice Research } \\
\text { Database } \\
3744 \text { cases, } 21757 \text { controls }\end{array}$ & $\begin{array}{l}\text { Aged } 5-79 \text { years with ICD code for asthma or } \\
\text { COPD with } \geq 1 \text { prescription for ICS and/or } \\
\text { OCS; or with no exposure to corticosteroids } \\
\text { From there } 65779 \text { individuals aged } 5- \\
17 \text { years identified to form base population for } \\
\text { study } \\
\text { Selection of cases: Patients with } 1 \text { st-time } \\
\text { diagnosis ICD-8 bone fracture; male } 65.6 \% \\
\text { Selection of controls: Up to } 6 \text { control subjects } \\
\text { selected per case, matched on age, gender, } \\
\text { general practice attended, calendar time and } \\
\text { years of history in GPRD; male } 64.9 \%\end{array}$ & $\begin{array}{l}76.2 \% \text { BDP } \\
21.7 \% \text { BUD } \\
2.1 \% \text { FP }\end{array}$ \\
\hline Sosa et $a f^{28}$ & Cross-sectional study & $\begin{array}{l}\text { BMD; Fracture } \\
\text { risk }\end{array}$ & $\begin{array}{l}\text { Canary Islands, Spain } \\
105 \text { cases; } 133 \text { controls }\end{array}$ & $\begin{array}{l}\text { Selection of cases: Women suffering from } \\
\text { stable bronchial asthma, treated with ICS } \\
\geq 1 \text { year, and who did not receive oral or } \\
\text { parenteral steroids. Mean age: } 53.0 \text { years, } \\
\text { number of menopausal subjects n (\%): } 65 \\
(61.9) \\
\text { Selection of controls: weight-matched women, } \\
\text { no asthma and no steroids. Controls were } \\
\text { usually friends or neighbours of the patients. } \\
\text { Mean age: } 49.7 \text { years, number of menopausal } \\
\text { subjects n (\%): } 74(57.8)\end{array}$ & $\begin{array}{l}\text { ICS formulations } \\
\text { not specified }\end{array}$ \\
\hline $\begin{array}{l}\text { Van Staa } \\
\text { et } a \text { P }^{9}\end{array}$ & $\begin{array}{l}\text { Population-based cohort } \\
\text { study/nested case-control } \\
\text { analysis }\end{array}$ & Fracture risk & $\begin{array}{l}\text { UK General Practice Research } \\
\text { Database (GPRD) } \\
\text { Cohort: ICS users: } 97387 \\
\text { Bronchodilators only: } 70984 \\
\text { Controls: } 345758 \\
\text { Fracture cases: } 23984 \text {; Controls: } \\
23984\end{array}$ & $\begin{array}{l}\text { Children aged } 4-17 \text { years old, on ICS. } 3 \text { study } \\
\text { groups: selection of cases: non-vertebral } \\
\text { fracture. Male } 61 \%, 8856(36.9 \%) \text { aged } 4- \\
9 \text { years, } 8496(35.4 \%) \text { aged } 10-13 \text { years, } \\
6632(27.7 \%) \text { aged } 14-17 \text { years } \\
\text { Selection of controls: for each fracture case, } \\
\text { one control patient randomly selected, } \\
\text { matched by age, sex, GP practice and } \\
\text { calendar time. Male } 61 \%, 8861(36.9 \%) \text { aged }\end{array}$ & BDP, BUD, FP \\
\hline
\end{tabular}

Continued 
Table 1 Continued

\begin{tabular}{|c|c|c|c|c|c|}
\hline \multicolumn{6}{|c|}{ (B) Observational studies } \\
\hline \multirow[t]{2}{*}{ Study } & \multirow[t]{2}{*}{ Design } & \multirow[t]{2}{*}{$\begin{array}{l}\text { Adverse } \\
\text { effects } \\
\text { measured } \\
\end{array}$} & \multirow[t]{2}{*}{$\begin{array}{l}\text { Data source and number of } \\
\text { patients }\end{array}$} & $\begin{array}{l}\text { Selection of patients: asthma definition } \\
\text { and patient characteristics (or selection of } \\
\text { cases and controls) }\end{array}$ & \multirow[t]{2}{*}{ Type of ICS } \\
\hline & & & & $\begin{array}{l}4-9 \text { years, } 8497(35.4 \%) \text { aged } 10-13 \text { years, } \\
6626(27.6 \%) \text { aged } 14-17 \text { years }\end{array}$ & \\
\hline \multirow[t]{2}{*}{$\begin{array}{l}\text { Wisniewski } \\
\text { et } a \beta^{\beta 0}\end{array}$} & \multirow[t]{2}{*}{ Cross-sectional study } & \multirow[t]{2}{*}{ BMD } & \multirow{2}{*}{$\begin{array}{l}\text { Asthma register and local general } \\
\text { practices in Nottingham, UK } \\
47 \text { cases; } 34 \text { controls }\end{array}$} & $\begin{array}{l}\text { Selection of cases: aged } 20-40 \text { years with } \\
\text { documented history of asthma: }\end{array}$ & \multirow[t]{2}{*}{ BDP, BUD } \\
\hline & & & & $\begin{array}{l}\text { Group 1: asthmatics using inhaled } \beta_{2} \text {-agonist } \\
\text { only. Males } 56 \% \text {, mean age: men } 30.3 \text { years; } \\
\text { women } 25.6 \text { years, mean FEV } 1 \text { (litres): men } \\
3.87 \text {; women } 3.13 \\
\text { Group } 2 \text { : ICS use } \geq 5 \text { years with no systemic } \\
\text { steroids in the past } 6 \text { months. Males } 40 \% \text {, } \\
\text { mean age: men } 32.3 \text { years; women } 32 \text { years, } \\
\text { mean FEV } \text { (litres): men } 3.40 \text {; women } 2.83\end{array}$ & \\
\hline \multirow[t]{3}{*}{ Yanik et $a \beta^{\beta 1}$} & \multirow[t]{3}{*}{ Observational } & \multirow[t]{3}{*}{ BMD } & \multirow{3}{*}{$\begin{array}{l}\text { Pulmonology outpatient clinic at Fatih } \\
\text { University Faculty of Medicine, } \\
\text { Ankara, Turkey } \\
46 \text { cases, } 60 \text { controls }\end{array}$} & $\begin{array}{l}\text { Selection of cases: Regular ICS use } \\
\geq 12 \text { months) as defined by The Global } \\
\text { Initiative for Asthma (GINA) criteria }\end{array}$ & \multirow[t]{3}{*}{$\mathrm{BDP}, \mathrm{BUD}, \mathrm{FP}$} \\
\hline & & & & $\begin{array}{l}\text { Mean age: } 62.5 \text { years, male } 0 \%, \% F V_{1} \\
\text { predicted: } 83.1 \text {, All cases were } \\
\text { postmenopausal }\end{array}$ & \\
\hline & & & & $\begin{array}{l}\text { Selection of controls: healthy postmenopausal } \\
\text { females. Mean age: } 63 \text { years }\end{array}$ & \\
\hline
\end{tabular}


Table 2 Study validity and outcomes (bone mineral density and fractures) in children

\begin{tabular}{|c|c|c|c|c|c|c|c|}
\hline \multicolumn{8}{|c|}{ (A) RCTs of inhaled corticosteroids-children } \\
\hline $\begin{array}{l}\text { CAMP/Kelly } \\
\text { et } a 1^{15} 32\end{array}$ & $\begin{array}{l}\text { Permuted blocks, } \\
\text { stratified }\end{array}$ & Adequate & Adequate & $\begin{array}{l}\text { Height recorded at every visit; } \\
\text { BMD once every year }\end{array}$ & $\begin{array}{l}\text { Fracture rate (adjusted for } \\
\text { age, ethnic group, sex, } \\
\text { clinic, base line duration, } \\
\text { skin-test reactivity and } \\
\text { asthma severity): } \\
\text { BUD: } 5.7 \text { per } 100 \\
\text { person-years } \\
\text { Placebo: } 5.1 \text { per } 100 \\
\text { person-years } \\
\text { p=0.59 } \\
\text { Mean difference in BMD } \\
\text { (ICS vs placebo): } \\
\text { Females: }-0.001 \text { (derived } \\
\text { SE } 0.0016 \text { ) } \\
\text { Male:-0.003 (derived SE } \\
0.0014 \text { ) }\end{array}$ & $11 \%$ & $5 \%$ \\
\hline Roux et $a l^{19}$ & $\begin{array}{l}\text { Central Block } \\
\text { randomisation with } \\
\text { gender } \\
\text { stratification }\end{array}$ & & $\begin{array}{l}\text { Largely open. } \\
\text { Analysis of } \\
\text { DEXA scans } \\
\text { blinded }\end{array}$ & $\begin{array}{l}\text { Lumber spine and femoral neck } \\
\text { BMD (DEXA) during run-in and } \\
6,12 \text { and } 24 \text { months. Adjusted } \\
\text { for age, height, weight, baseline } \\
\text { BMD, gender and measuring } \\
\text { device }\end{array}$ & $\begin{array}{l}\text { Mean difference in lumbar } \\
\text { spine BMD for FP vs } \\
\text { control: } 0.012 \text { (SE } \\
0.0073 \text { ); values calculated } \\
\text { from \% change in } \\
\text { manuscript }\end{array}$ & $23 \%$ & $4 \%$ \\
\hline $\begin{array}{l}\text { Turpeinen } \\
\text { et } a^{\text {PO }}\end{array}$ & Block & Unclear & $\begin{array}{l}\text { Blinded for } \\
\text { budesonide and } \\
\text { placebo arms }\end{array}$ & $\begin{array}{l}\text { BMD of } L 1-4 \text { measured by } \\
\text { radiologist using DEXA at } \\
\text { baseline and at } 18 \text { months }\end{array}$ & $\begin{array}{l}\text { Mean change in lumbar } \\
\text { spine BMD: } \\
\text { Budesonide for } \\
12 \text { months } 0.023 \text { (SD } \\
0.022 \text { ) } \\
\text { Placebo for } 12 \text { months } \\
0.029 \text { (SD } 0.022) \\
\text { DSCG: } 0.034 \text { (SD 0.022) }\end{array}$ & $20 \%$ & $3 \%$ \\
\hline
\end{tabular}

BUD, budesonide; DEXA, dual-energy X-ray absorptiometry; DSCG, disodium cromoglicate; FP, fluticasone propionate. 
Table 2 Continued

(B) Observational studies of bone mineral density and fractures-children

\begin{tabular}{|c|c|c|c|c|c|c|}
\hline Study & Ascertainment of BMD & $\begin{array}{l}\text { Ascertainment of } \\
\text { exposure }\end{array}$ & Definition of ICS use & Adjustments & ICS exposure & BMD $(\mathbf{g} / \mathbf{c m})$ \\
\hline $\begin{array}{l}\text { Agertoft and } \\
\text { Pedersen }^{21}\end{array}$ & $\begin{array}{l}\text { DEXA scan at one visit, performed by } \\
\text { same investigator blinded to treatment } \\
\text { group }\end{array}$ & $\begin{array}{l}\text { Compliance } \\
\text { checked: Good } \\
\text { Duration: } \\
\text { Mean } 1603 \text { days }\end{array}$ & $\begin{array}{l}\text { Asthmatic children with } \\
\text { ICS use continuously } \\
\text { for } \geq 3 \text { years } \\
\text { Type of inhaler: MDI; } \\
\text { Turbuhaler } \\
\text { Type of steroid: BUD }\end{array}$ & $\begin{array}{l}\text { Log of accumulated } \\
\text { dose of BUD; gender; } \\
\text { age }\end{array}$ & $\begin{array}{l}\text { Mean ICS BUD } \\
\text { dose } 504 \mu \mathrm{g} \\
\text { (daily) }\end{array}$ & $\begin{array}{l}\text { Mean BMD: } \\
\text { BUD group: } 0.92 \\
\text { Control group: } 0.92\end{array}$ \\
\hline Allen et $a R^{2}$ & $\begin{array}{l}\text { DEXA scan at baseline and again at 9- } \\
20 \text { months later. Value for } 12 \text {-month } \\
\text { time point calculated with all outcomes }\end{array}$ & $\begin{array}{l}\text { Compliance } \\
\text { checked: Adequate } \\
\text { Duration of } \\
\text { follow-up: 9- } \\
20 \text { months }\end{array}$ & $\begin{array}{l}\text { Type of inhaler: } \\
\text { Spacer, Turbohaler } \\
\text { Type of steroid: BDP, } \\
\text { BUD }\end{array}$ & $\begin{array}{l}\text { Age; height; weight; } \\
\text { dose of inhaled } \\
\text { corticosteroid }\end{array}$ & $\begin{array}{l}\text { Mean ICS dose } \\
0.67 \pm 0.48 \mathrm{mg} / \\
\mathrm{m}^{2} / \text { day }\end{array}$ & $\begin{array}{l}\text { Change in mean } \\
\text { vertebral BMD }(S D) \\
\text { over } 12 \text { months: } \\
\text { ICS group }(n=47) \text { : } \\
0.03 \pm 0.03 \\
\text { Control group } \\
(n=9): 0.06 \pm 0.04 \\
\text { p: }<0.025\end{array}$ \\
\hline $\begin{array}{l}\text { Bahceciler } \\
\text { et } a f^{3}\end{array}$ & $\begin{array}{l}\text { Anteroposterior (AP) spine (L2-4) by } \\
\text { DEXA scan }\end{array}$ & $\begin{array}{l}\text { Compliance: not } \\
\text { reported } \\
\text { Follow-up: } 13.0 \\
\pm 9.8 \text { months }\end{array}$ & $\begin{array}{l}\text { Use of BUD as MDI } \\
\geq 6 \text { months }\end{array}$ & None & $\begin{array}{l}\text { ICS Mean daily } \\
\text { dose (SD): } 419 \\
\pm 154 \mu \mathrm{g} \\
\text { Control }\end{array}$ & $\begin{array}{l}\text { Mean lumbar spine } \\
\text { BMD: } \\
\text { ICS group: } 0.593 \\
\text { (SD 0.122) } \\
\text { Mean lumbar spine } \\
\text { BMD: } 0.579 \text { (SD } \\
0.156)\end{array}$ \\
\hline Harris et $a^{25}$ & Lumbar spine by DEXA & $\begin{array}{l}\text { Compliance } \\
\text { checked: not } \\
\text { reported } \\
\text { Duration of } \\
\text { follow-up: } 3.5 \\
\pm 2.4 \text { years }\end{array}$ & $\begin{array}{l}\text { Stratified by treatment } \\
\text { in past } 6 \text { months } \\
\text { Type of inhaler: Spacer } \\
\text { device } \\
\text { Type of steroid: BDP, } \\
\text { BUD, FP }\end{array}$ & Weight & 400-800 $\mu \mathrm{g} /$ day & $\begin{array}{l}\text { Mean lumbar spine } \\
\text { BMD (SD) } 0.68 \\
(0.07) \\
\text { Mean lumbar spine } \\
\text { BMD (SD) } \\
0.70(0.08) \\
\text { Mean lumbar spine } \\
\text { BMD (SD) } \\
0.67(0.08)\end{array}$ \\
\hline
\end{tabular}


Table 2 Continued

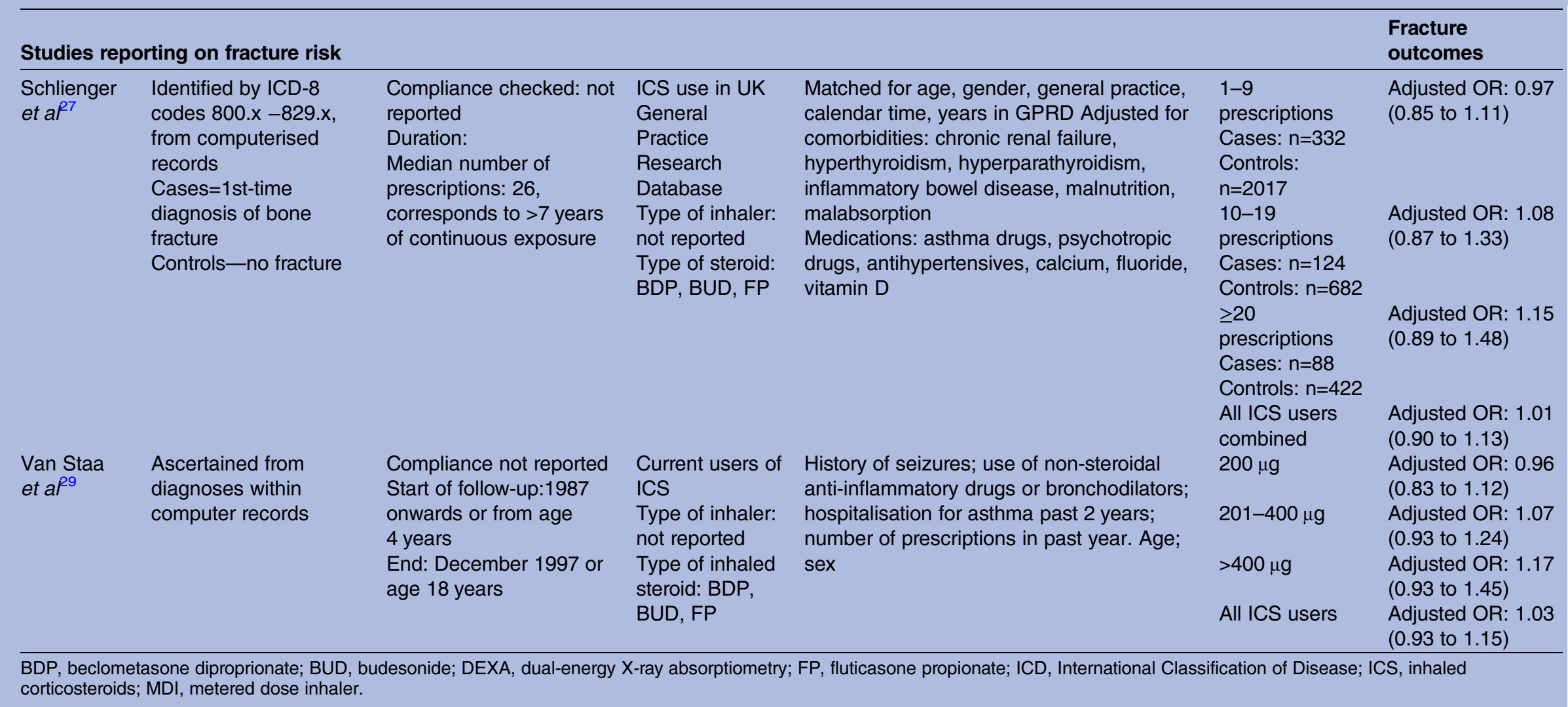


Table 3 Study validity and outcomes (bone mineral density and fractures) in adults

(a) RCTs of inhaled corticosteroids-adults

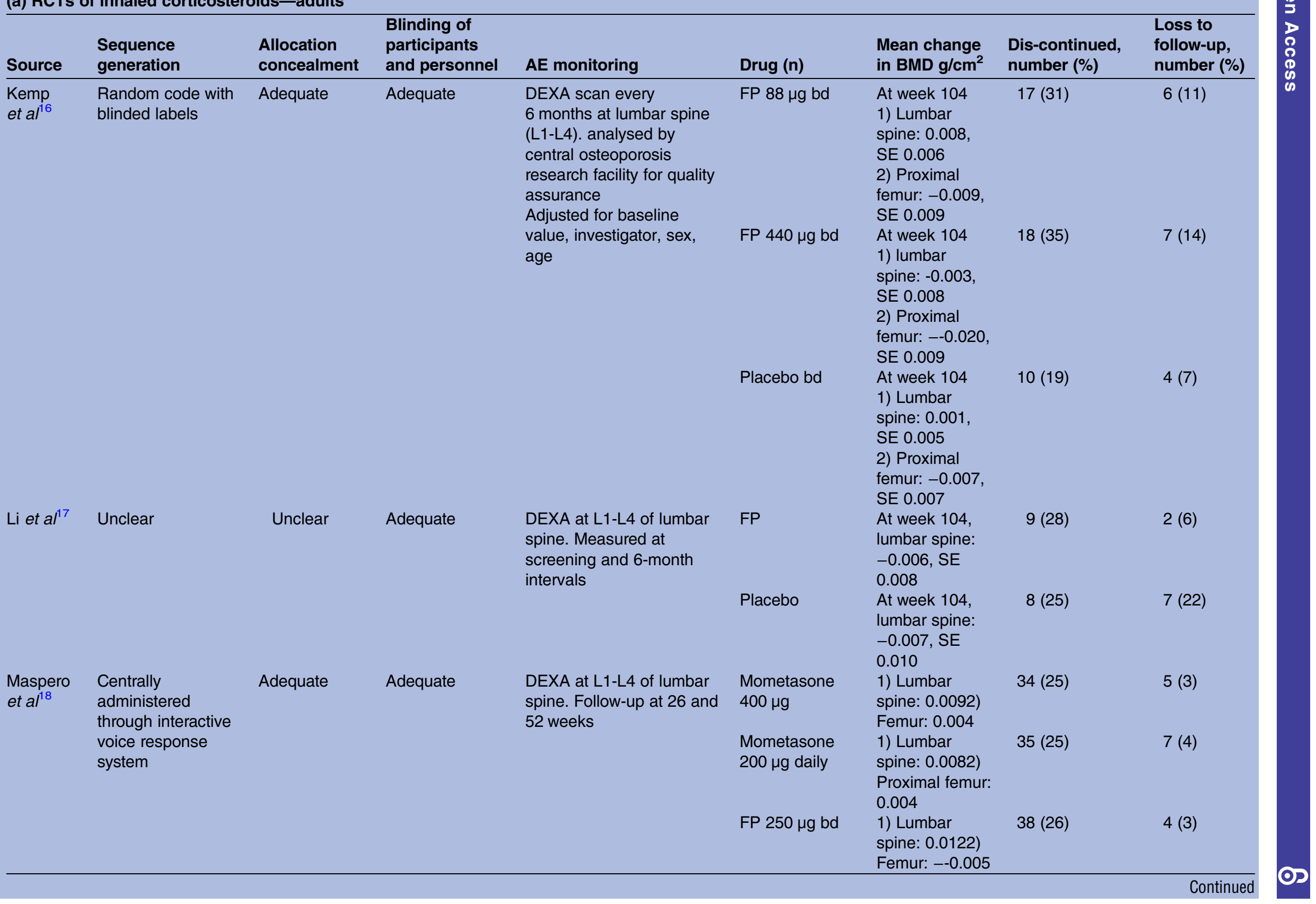


Table 3 Continued

(a) RCTs of inhaled corticosteroids-adults

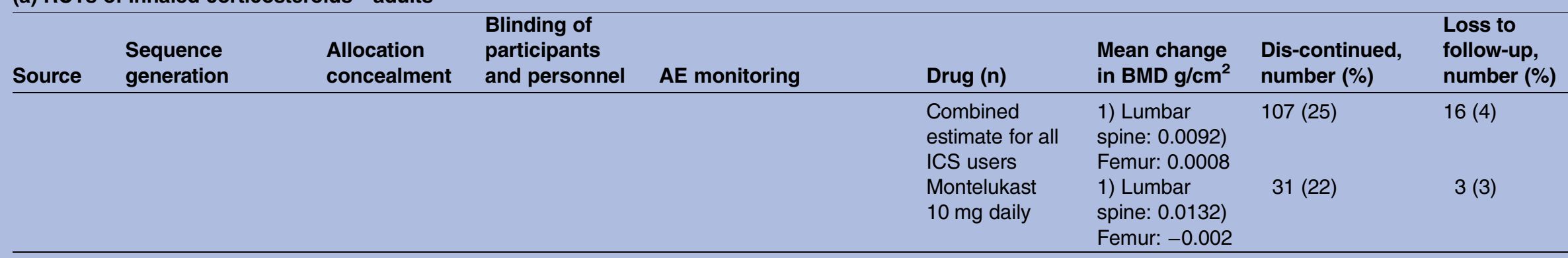

$\mathrm{AE}$, adverse event; bd, two times a day; DEXA, dual-energy X-ray absorptiometry; FP, fluticasone propionate; ICS, inhaled corticosteroids; RCT, randomised controlled trial.

\begin{tabular}{|c|c|c|c|c|c|c|}
\hline \multicolumn{7}{|c|}{ Observational studies of bone mineral density and fractures-adults } \\
\hline Study & $\begin{array}{l}\text { Ascertainment of BMD/ } \\
\text { fracture }\end{array}$ & $\begin{array}{l}\text { Ascertainment of } \\
\text { ICS exposure }\end{array}$ & Definition of ICS use & Adjustments & $\begin{array}{l}\text { ICS } \\
\text { exposure }\end{array}$ & $\begin{array}{l}\text { Results of BMD ( } \mathrm{g} / \\
\left.\mathrm{cm}^{2}\right) \text { and fractures }\end{array}$ \\
\hline $\mathrm{El}$ et $a^{R^{4}}$ & $\begin{array}{l}\text { DEXA lumbar spine (L1-4) and } \\
\text { femoral neck }\end{array}$ & $\begin{array}{l}\text { Compliance checked: } \\
\text { poor } \\
\text { Duration: } \\
\text { mean duration (SD) } \\
\text { (years): } 2.79 \pm 1.77\end{array}$ & $\begin{array}{l}\text { Regular ICS } \\
>6 \text { months } \\
\text { Type of inhaler: not } \\
\text { reported } \\
\text { Type of ICS: not } \\
\text { reported }\end{array}$ & Age & $\begin{array}{l}\text { Cases } \\
\text { Mean daily } \\
\text { ICS dose } \\
326.43 \mu \mathrm{g} \\
\text { Controls (no } \\
\text { exposure) }\end{array}$ & $\begin{array}{l}\text { Mean lumbar: } 0.925 \text {, } \\
\text { SD } 0.211 \\
\text { Mean femoral neck: } \\
0.746, \text { SD } 0.127 \\
\text { Mean lumbar: BMD: } \\
0.927, \text { SD } 0.229 \\
\text { Mean femoral neck: } \\
0.792, \text { SD } 0.097\end{array}$ \\
\hline $\begin{array}{l}\text { Johannes } \\
\text { et } a^{\text {R6 }}\end{array}$ & $\begin{array}{l}\text { Non-vertebral identified by } \\
\text { ICD- } 9 \text { codes and insurance } \\
\text { claim for fracture treatment } \\
\text { within } 2 \text { weeks }\end{array}$ & $\begin{array}{l}\text { Compliance checked: } \\
\text { not reported } \\
\text { Duration: } 1 \text { Year ICS } \\
\text { exposure }\end{array}$ & $\begin{array}{l}\text { ICS use from } \\
\text { pharmacy claims in } \\
\text { the } 365 \text { days before } \\
\text { index date. } \\
\text { Type of inhaler: not } \\
\text { reported } \\
\text { Type of steroid: BDP, } \\
\text { BUD, FP, flunisolone, } \\
\text { triamcinolone }\end{array}$ & $\begin{array}{l}\text { Demographics-age, sex, region, } \\
\text { time and season } \\
\text { Comorbidities-wide range of } \\
\text { cardiovascular, endocrine, } \\
\text { metabolic and musculoskeletal } \\
\text { conditions } \\
\text { Medications-oral corticosteroids, } \\
\text { bisphosphonates, statins, } \\
\text { anticonvulsants, oestrogen, } \\
\text { raloxifene, calcitonin } \\
\text { Healthcare utilisation for } \\
\text { underlying respiratory disease }\end{array}$ & $\begin{array}{l}1-167 \mu \mathrm{g} \\
168-504 \mu \mathrm{g} \\
505-840 \mu \mathrm{g} \\
>840 \mu \mathrm{g}\end{array}$ & $\begin{array}{l}\text { OR } 1.00 \\
95 \% \mathrm{Cl} 0.84 \text { to } 1.18 \\
\text { OR: } 1.02 \\
95 \% \mathrm{Cl} 0.83 \text { to } 1.26 \\
\text { OR: } 1.14 \\
95 \% \mathrm{Cl} 0.80 \text { to } 1.62 \\
0.99 \\
95 \% \mathrm{Cl} 0.66 \text { to } 1.50\end{array}$ \\
\hline Sosa et $a^{28}$ & $\begin{array}{l}\text { DEXA } \\
\text { lumbar spine (L2-L4) and } \\
\text { proximal femur }\end{array}$ & $\begin{array}{l}\text { Compliance: not } \\
\text { reported } \\
\text { Duration of follow up: }\end{array}$ & $\begin{array}{l}\text { ICS for >1 year } \\
\text { Type of inhaler: not } \\
\text { reported }\end{array}$ & Age & $\begin{array}{l}\text { Cases (dose } \\
\text { not } \\
\text { reported) }\end{array}$ & $\begin{array}{l}\text { Lumbar spine: } \\
0.960 ; 95 \% \text { Cl } 0.925 \\
\text { to } 0.995 \\
\text { Femoral neck:0.776; }\end{array}$ \\
\hline
\end{tabular}


Observational studies of bone mineral density and fractures-adults

\begin{tabular}{|c|c|c|c|c|c|c|}
\hline Study & $\begin{array}{l}\text { Ascertainment of BMD/ } \\
\text { fracture }\end{array}$ & $\begin{array}{l}\text { Ascertainment of } \\
\text { ICS exposure }\end{array}$ & Definition of ICS use & Adjustments & $\begin{array}{l}\text { ICS } \\
\text { exposure }\end{array}$ & $\begin{array}{l}\text { Results of BMD ( } \mathrm{g} / \\
\mathrm{cm}^{2} \text { ) and fractures }\end{array}$ \\
\hline & & $\begin{array}{l}\text { Median treatment } \\
\text { with ICS: } 10 \text { years }\end{array}$ & $\begin{array}{l}\text { Type of ICS: not } \\
\text { reported }\end{array}$ & & Controls & $\begin{array}{l}95 \% \mathrm{Cl} 0.750 \text { to } \\
0.802 \\
\text { Fractures: } 22 / 105 \\
(21.0 \%) \\
\text { Lumbar spine: } \\
0.991 ; 95 \% \mathrm{Cl} 0.960 \\
\text { to } 1.022 \\
\text { Femoral neck: } \\
0.780 ; 95 \% \mathrm{Cl} 0.758 \\
\text { to } 0.803 \\
\text { Fractures: } 9 / 133 \\
(7.0 \%)\end{array}$ \\
\hline $\begin{array}{l}\text { Wisniewski } \\
\text { et } a \beta^{\beta 0}\end{array}$ & $\begin{array}{l}\text { Posterior-anterior spine (L2-4), } \\
\text { lateral spine (body of L3) } \\
\text { measured by DEXA once. All } \\
\text { scans by same radiographer } \\
\text { (blinded) }\end{array}$ & $\begin{array}{l}\text { Compliance checked: } \\
\text { Adequate } \\
\text { Duration: } \\
\text { Median duration of } \\
\text { use of ICS (years) } \\
\text { Men: } 9.00 \\
\text { Women: } 6.29\end{array}$ & $\begin{array}{l}\text { ICS for > } 5 \text { years } \\
\text { Type of inhaler: } \\
\text { Metered dose inhaler } \\
-36 \text { patients; dry } \\
\text { powder inhaler-11 } \\
\text { patients } \\
\text { Type of ICS: BDP, } \\
\text { BUD }\end{array}$ & $\begin{array}{l}\text { age; weight; smoking; alcohol; } \\
\text { activity grade; asthma severity; } \\
\text { age at menarche; lifetime total } \\
\text { dose of oestrogen and } \\
\text { progesterone; prednisolone use }\end{array}$ & Cases & $\begin{array}{l}\text { Lumbar spine } \pm S D \\
\text { Men : } 1.28 \pm 0.13 ; \\
\text { Women: } 1.04 \pm 0.14 \\
\text { Femoral neck } \pm S D \text { : } \\
\text { Men : } 1.17 \pm 0.18 ; \\
\text { Women: } 1.09 \pm 0.14 \\
\text { Vertebral fractures } \\
\text { overall: } 2 / 47\end{array}$ \\
\hline & & & & & $\begin{array}{l}\text { Controls (No } \\
\text { exposure) }\end{array}$ & $\begin{array}{l}\text { Lumbar spine } \pm S D \\
\text { Men: } 1.21 \pm 0.17 ; \\
\text { Women: } 1.25 \pm 0.12 \\
\text { Femoral neck } \pm S D \text { : } \\
\text { Men : } 1.04 \pm 0.14 ; \\
\text { Women: } 1.10 \pm 0.14 \\
\text { Vertebral fractures } \\
\text { overall: } 6 / 34\end{array}$ \\
\hline Yanik et $a \beta^{\beta 1}$ & $\begin{array}{l}\text { DEXA lumbar spine and hip } \\
\text { (femoral neck and trochanter). } \\
\text { Patient-reported history of } \\
\text { fractures }\end{array}$ & $\begin{array}{l}\text { Compliance checked: } \\
\text { Adequate } \\
\text { Duration of } \\
\text { Follow-up: } 4.3 \\
\pm 2.6 \text { years }\end{array}$ & $\begin{array}{l}\text { Regular ICS } \\
>12 \text { months } \\
\text { Type of inhaler: Not } \\
\text { reported } \\
\text { Type of ICS: } \\
\text { BDP, BUD, FP }\end{array}$ & None & $\begin{array}{l}\text { Cases } \\
\text { (total) } \\
\text { Mean daily } \\
\text { ICS dose } \\
(\mu \mathrm{g})(\mathrm{SD}): \\
324.9 \pm 121.8 \\
\text { Controls }\end{array}$ & $\begin{array}{l}\text { Lumbar spine } \pm S D \\
0.95 \pm 0.29 \\
\text { Femoral neck } \pm S D \\
0.83 \pm 0.12 \\
\text { Atraumatic vertebral } \\
\text { fractures: } 4(8.6 \%) \\
\text { Lumbar spine } \pm S D \\
0.88 \pm 0.14 \\
\text { Femoral neck } \pm S D \\
0.74 \pm 0.23 \\
\text { Atraumatic vertebral } \\
\text { fracture: } 6(10 \%)\end{array}$ \\
\hline
\end{tabular}


Figure 2 Fracture risk, ICS use versus non-use. ICS, inhaled corticosteroids.

Fractures Children

\begin{tabular}{|c|c|c|c|c|c|}
\hline Study or Subgroup & Weight & $\begin{array}{l}\text { Odds Ratio } \\
\text { IV, Random, } 95 \% \mathrm{CI}\end{array}$ & & \multicolumn{2}{|l|}{$\begin{array}{l}\text { Odds Ratio } \\
\text { IV, Random, } 95 \% \mathrm{Cl}\end{array}$} \\
\hline Schlienger 2004 & $47.0 \%$ & $1.01[0.90,1.13]$ & & & \\
\hline Van Staa 2004 & $53.0 \%$ & $1.03[0.93,1.15]$ & & & \\
\hline Total $(95 \% \mathrm{CI})$ & $100.0 \%$ & $1.02[0.94,1.10]$ & & & \\
\hline \multicolumn{3}{|c|}{$\begin{array}{l}\text { Heterogeneity: } \mathrm{Tau}^{2}=0.00 ; \mathrm{Chi}^{2}=0.06, \mathrm{df}=1(P=0.80) ;\left.\right|^{2}=0 \% \\
\text { Test for overall effect: } Z=0.52(P=0.60)\end{array}$} & 0.7 & $\begin{array}{lcc}0.85 & 1 & 1.2 \\
\text { ICS safe } & \text { ICS harmful }\end{array}$ & 1.5 \\
\hline
\end{tabular}

Fractures Adults

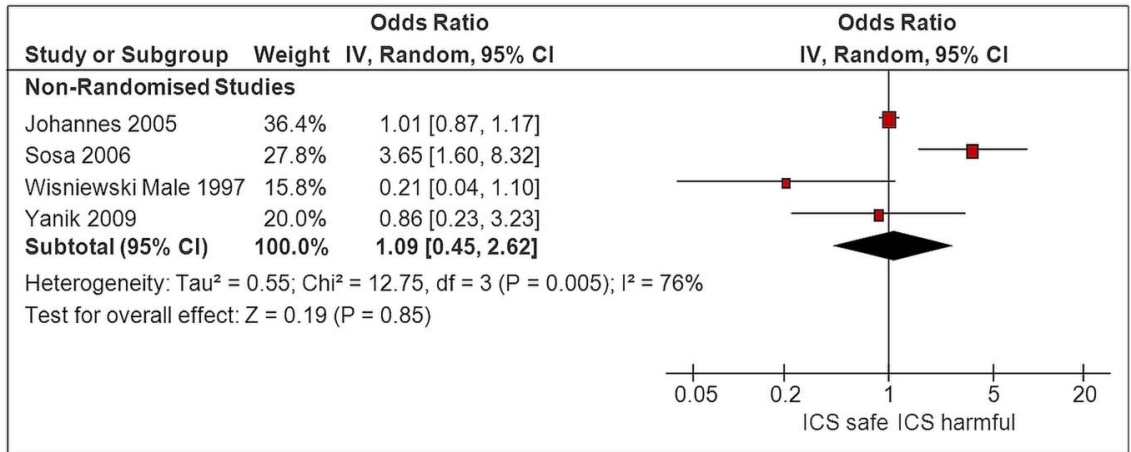

significantly elevated at higher dose levels, with one study demonstrating an OR of 1.15 (0.89 to 1.48) for children with $\geq 20$ prescriptions, ${ }^{27}$ and the other study reporting an OR of 1.17 (0.93 to 1.45) for children using a daily dose of $>400 \mu \mathrm{g}$ beclomethasone dipropionate equivalents. ${ }^{29}$

No consistent association between ICS use and fracture risk in adults was seen in the pooled estimate from four observational studies (overall OR 1.09, 95\% CI 0.45 to 2.62; figure 2). ${ }^{26283031}$ There was substantial heterogeneity in this meta-analysis $\left(\mathrm{I}^{2}=76 \%\right)$, with Sosa's study reporting significantly increased fracture risk, ${ }^{28}$ while the others did not.

However, we judged a study by Sosa $e t a l^{28}$ to be at high risk of bias because the control group consisted of relatives and neighbours of patients, the type of ICS was not reported and there were no statistical adjustments for confounders. In this data set, Johannes $e t a l^{26}$ was the only study reporting fractures according to dose, but this did not demonstrate any consistent trend towards elevated risk at higher doses.

\section{Lumbar spine BMD}

Three RCTs and three observational studies reported on comparative change at the lumbar spine in children. 151920222325 (figure 3) ICS use was not associated with significant reductions in BMD as compared to controls in RCTs (mean difference $-0.0018 \mathrm{~g} / \mathrm{cm}^{2} ; 95 \%$ CI -0.0051 to $0.0015 \mathrm{~g} / \mathrm{cm}^{2} ; \mathrm{I}^{2}=46 \%$ ) or observational studies (mean difference $-0.0075 \mathrm{~g} / \mathrm{cm}^{2} ; 95 \%$ CI -0.044 to $0.028 \mathrm{~g} / \mathrm{cm}^{2} ; \mathrm{I}^{2}=42 \%$ ). There was no clear signal of dose responsiveness in one observational study that separated

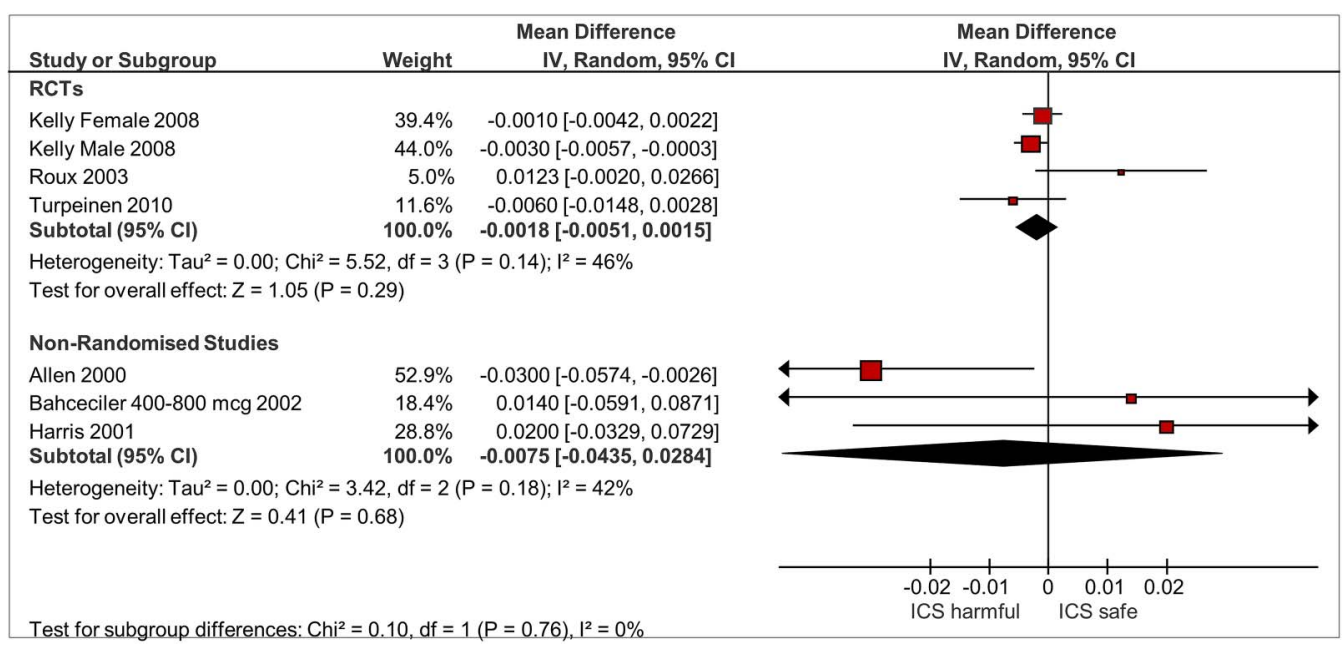

Figure 3 BMD in lumbar spine children, ICS use versus non-use. BMD, bone mineral density; ICS, inhaled corticosteroids. 
Spine Adults

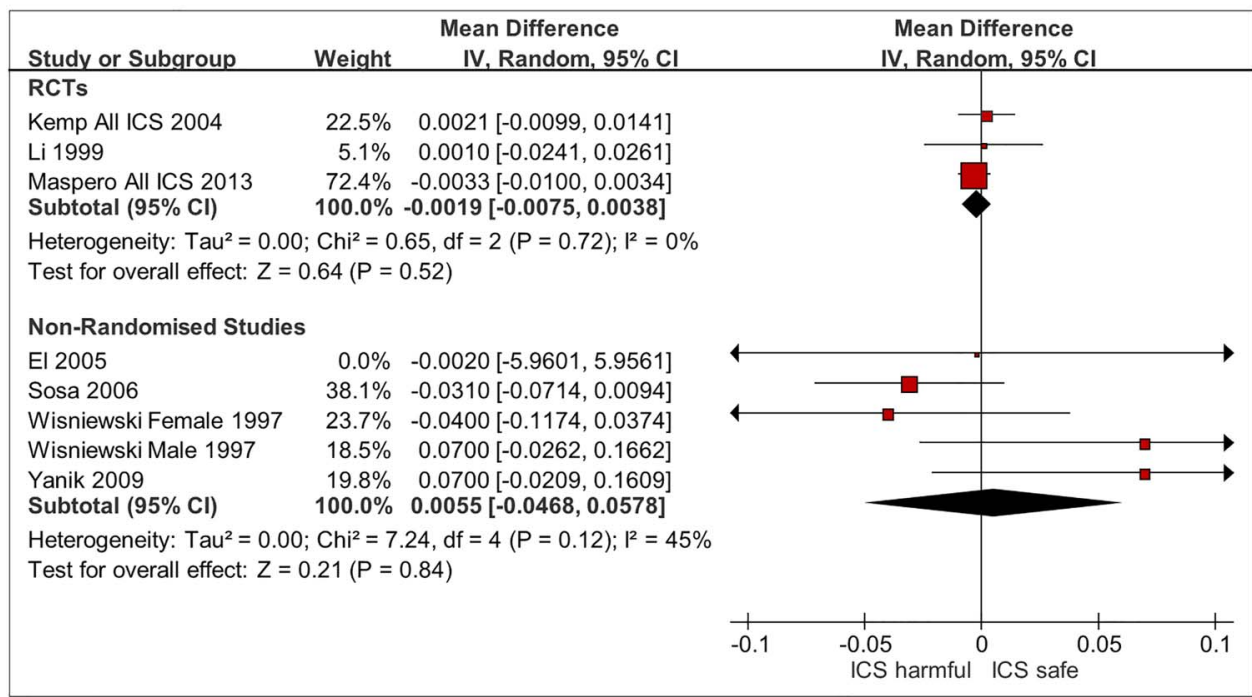

Femur/Hip Adults

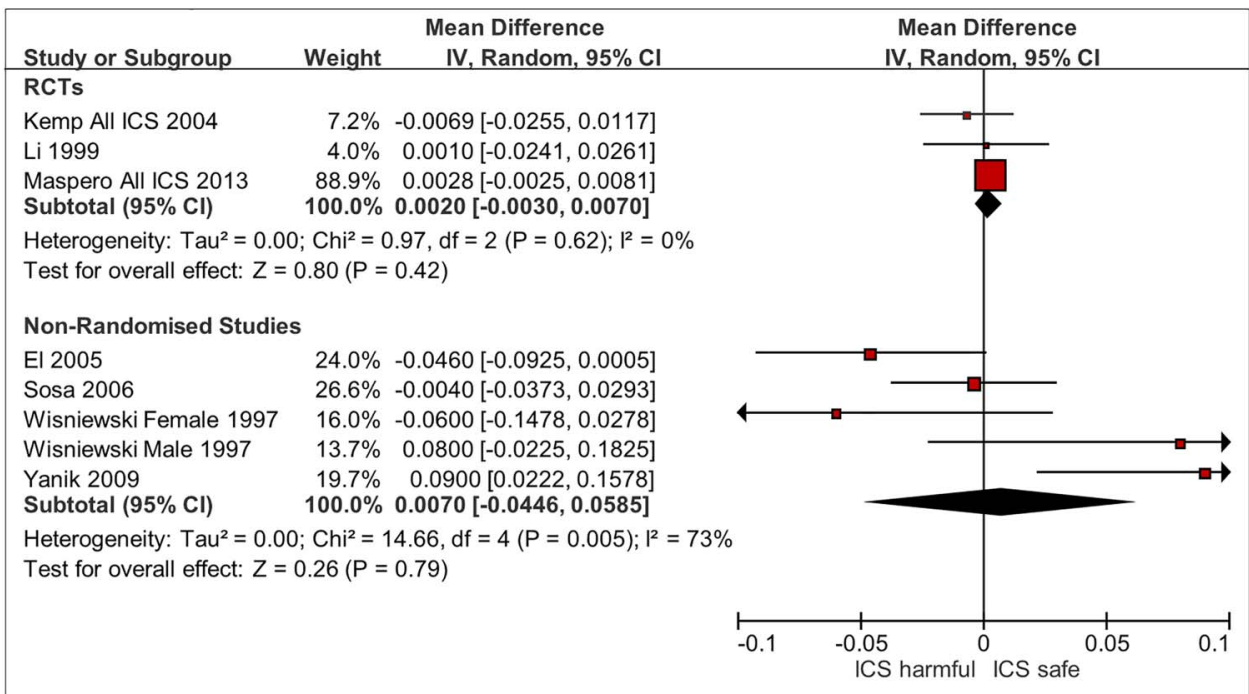

Figure 4 BMD in adults, ICS use versus non-use. BMD, bone mineral density; ICS, inhaled corticosteroids; RCT, randomised controlled trial.

participants into different dose levels, ${ }^{25}$ whereas one RCT suggested that longer term users of budesonide with greater cumulative doses had lower BMD compared to those who received lower cumulative doses. ${ }^{20}$

Three RCTs and four observational studies reported on comparative change in BMD at the lumbar spine in adults (figure 4). ${ }^{16-18} 24283031$ ICS use was not associated with significant reductions in BMD as compared to controls in RCTs (mean difference $-0.0019 \mathrm{~g} / \mathrm{cm}^{2}$; $95 \%$ CI -0.0075 to $0.0038 \mathrm{~g} / \mathrm{cm}^{2} ; \mathrm{I}^{2}=0 \%$ ) or observational studies (mean difference $-0.0055 \mathrm{~g} / \mathrm{cm}^{2} ; 95 \%$ CI -0.047 to $0.058 \mathrm{~g} / \mathrm{cm}^{2} ; \mathrm{I}^{2}=45 \%$ ).

\section{Femur/hip BMD for adults}

There were three RCTs and four observational studies reporting comparative change in BMD at the femur or hip in adults (figure 4). ${ }^{16-18} 24283031$ ICS use was not associated with significant reductions in BMD as compared to controls in RCTs (mean difference $0.0020 \mathrm{~g} / \mathrm{cm}^{2} ; 95 \%$ CI -0.0030 to $0.0070 \mathrm{~g} / \mathrm{cm}^{2} ; \mathrm{I}^{2}=0 \%$ ) or observational studies (mean difference $0.0070 \mathrm{~g} / \mathrm{cm}^{2}$; $95 \%$ CI -0.045 to $0.059 \mathrm{~g} / \mathrm{cm}^{2} ; \mathrm{I}^{2}=73 \%$ ).

There was sparse data comparing different ICS molecules head to head. Ferguson et $a l^{14}$ measured lumbar spine BMD and reported a non-significant finding between children randomised to fluticasone propionate $100 \mu \mathrm{g}$ twice daily as compared to budesonide, mean difference $0.0075 \mathrm{~g} / \mathrm{cm}^{2}$ (95\% CI -0.033 to $\left.0.048 \mathrm{~g} / \mathrm{cm}^{2}\right)$. Maspero conducted a five arm trial that included mometasone and fluticasone propionate in adults. There were no significant differences in lumbar spine and femur BMD between the two compounds at the end of the trial. ${ }^{18}$ 
We did not proceed to constructing a funnel plot for detection of publication bias because we had less than 10 studies in the meta-analysis of each outcome, and there was substantial heterogeneity.

\section{DISCUSSION}

We focused our systematic review of RCTs and observational studies on skeletal adverse effects of ICS in patients with asthma. We did not find convincing evidence of increased fracture risk with ICS use in adults or children. Equally, there was no consistent evidence of any significant detrimental relationship between ICS use and BMD at the lumbar spine (in adults and children) or femur (in adults). There was insufficient data for us to detect any dose-response relationship, or to judge any potential differences between the available ICS molecules.

Our findings should be contrasted with those of other recent published reviews. There have been at least four systematic reviews evaluating fractures or BMD in ICS users, with two earlier reviews demonstrating a significant reduction in BMD but no definite impact on fractures. ${ }^{53}$ The most recent meta-analyses have identified a small but statistically significant dose-related increase in risk of fracture associated with ICS use in patients with COPD. ${ }^{6}{ }^{7}$ Our findings differ from these other reviews as we have specifically focused on ICS use in patients with asthma. Here, we used very rigid selection criteria in an attempt to exclude patients with COPD from our meta-analysis.

The deleterious effects of ICS on BMD seen in previous meta-analyses could be explained in part by the higher prevalence of smoking in patients with COPD as previous studies have shown that smoking has a harmful effect on $\mathrm{BMD}$, and increasing fracture risk. ${ }^{8}$ In addition, as a group, patients with asthma are likely to be younger and to have fewer comorbidities than those with COPD which may impact on BMD and fracture risk. Recent research indicates that multimorbidity (including cachexia and low-grade systemic inflammation) is often seen in patients with COPD, ${ }^{9}$ and it is conceivable that these factors may have a further negative impact on bone formation that accentuate the risks of ICS in COPD.

ICS therapy may have a positive impact on bone density through reduction of chronic inflammation and avoidance of need for acute short courses of oral corticosteroids during exacerbations. In addition, ICS may allow better control of asthma in patients such that they become more active, thereby slowing or preventing steroid-induced osteoporosis through the beneficial effects of physical activity on BMD. Bone mass can also be influenced by a wide range of other factors (such as nutrition, genetic make-up, endocrine status and amount of physical exercise), ${ }^{1}$ and ICS may therefore not be the most important influence on bone density in patients with asthma.

There are a number of limitations to our systematic review. Our search was limited to English language articles. Although, studies have attempted to assess skeletal adverse effects in many different ways, we have limited our review to clinically meaningful outcomes such as BMD in $\mathrm{g} / \mathrm{cm}^{2}$ at lumbar spine and femur, and fractures. We did not have sufficient data from the primary studies for us to conduct meaningful analyses on different combinations of drug compounds, inhaler devices and dosage regimens. Some of the included studies were published more than a decade ago, and advances in asthma care may have made their findings less applicable to current-day patients. We recognise that there is potential for risk of bias (stemming from substantial loss to follow-up for BMD measurements) within this data set. Hence, we are unable to interpret the effects of ICS in very long-term use of ICS over a decade or more.

Our systematic review demonstrates that there is no consistent evidence of serious skeletal harm from use of ICS. Although there are intrinsic limitations to the evidence, we believe that our systematic review provides some reassurance to patients and prescribers of ICS. Our findings enables ICS users to judge the benefits and harms of their medication in a more accurate manner and helps to address concerns and uncertainty surrounding the exact risk of skeletal adverse effects.

Contributors YKL and AMW conceptualised the review and obtained funding. YKL, DG, MT, PB and AMW selected studies and abstracted the data. YKL carried out the synthesis of the data and wrote the manuscript with critical input from all authors. YKL acts as guarantor for the paper.

Funding This manuscript presents a systematic review commissioned by Asthma UK (AUK-PG-2012-181), and we are grateful to the Asthma UK Research team for their guidance. The views expressed in this paper are those of the authors.

Competing interests None declared.

Provenance and peer review Not commissioned; externally peer reviewed.

Data sharing statement No additional data are available.

Open Access This is an Open Access article distributed in accordance with the Creative Commons Attribution Non Commercial (CC BY-NC 4.0) license, which permits others to distribute, remix, adapt, build upon this work noncommercially, and license their derivative works on different terms, provided the original work is properly cited and the use is non-commercial. See: http:// creativecommons.org/licenses/by-nc/4.0/

\section{REFERENCES}

1. Ricciardolo FLM. The treatment of asthma in children: inhaled corticosteroids. Pulm Pharmacol Ther 2007;20:473-82.

2. British-Thoracic-Society/Scottish-Intercollegiate-Guidelines-Network. British Guideline on the Management of Asthma. Thorax 2008;63 (Suppl 4):iv1-121.

3. National-Asthma-Education-and-Prevention-Program. Expert Panel Report 3 (EPR-3): guidelines for the Diagnosis and Management of Asthma-Summary Report 2007. J Allergy Clin Immunol 2007;120(5 Suppl):S94-138.

4. Rossi GA, Cerasoli F, Cazzola M. Safety of inhaled corticosteroids: room for improvement. Pulm Pharmacol Ther 2007;20:23-35.

5. Jones A, Fay JK, Burr M, et al. Inhaled corticosteroid effects on bone metabolism in asthma and mild chronic obstructive pulmonary disease. Cochrane Database Syst Rev 2002;(1):CD003537.

6. Loke YK, Cavallazzi R, Singh S. Risk of fractures with inhaled corticosteroids in COPD: systematic review and meta-analysis of randomised controlled trials and observational studies. Thorax 2011;66:699-708. 
7. Weatherall M, James K, Clay J, et al. Dose-response relationship for risk of non-vertebral fracture with inhaled corticosteroids. Clin Exp Allergy 2008;38:1451-8.

8. Law MR, Hackshaw AK. A meta-analysis of cigarette smoking, bone mineral density and risk of hip fracture: recognition of a major effect. BMJ 1997;315:841-6.

9. Vanfleteren LE, Spruit MA, Groenen M et al. Clusters of comorbidities based on validated objective measurements and systemic inflammation in patients with chronic obstructive pulmonary disease. Am J Respir Crit Care Med 2013;187:728-35.

10. Loke YK, Golder SP, Vandenbroucke JP. Comprehensive evaluations of the adverse effects of drugs: importance of appropriate study selection and data sources. Ther Adv Drug Saf 2011;2:59-68.

11. Ioannidis JP, Trikalinos TA. The appropriateness of asymmetry tests for publication bias in meta-analyses: a large survey. CMAJ 2007;176:1091-6.

12. Higgins JPT, Deeks JJ, Altman DG. Special topics in statistics. In: Higgins JPT, Green S, eds. Cochrane handbook for systematic reviews of interventions. Chichester, UK: John Wiley \& Sons, 2008. Chapter 16, pp 481-529.

13. Higgins JPT, Deeks JJ, Altman DG. Analysing data and undertaking meta-analyses. In: Higgins JPT, Green S, eds. Cochrane handbook for systematic reviews of interventions. Chichester, UK: John Wiley \& Sons, 2008. Chapter 9, pp 243-296.

14. Ferguson AC, Van Bever HP, Teper AM, et al. A comparison of the relative growth velocities with budesonide and fluticasone propionate in children with asthma. Respir Med 2007;101:118-29.

15. Kelly HW, Van Natta ML, Covar RA, et al. Effect of long-term corticosteroid use on bone mineral density in children: a prospective longitudinal assessment in the childhood Asthma Management Program (CAMP) study. Pediatrics 2008;122:e53-61.

16. Kemp JP, Osur S, Shrewsbury SB, et al. Potential effects of fluticasone propionate on bone mineral density in patients with asthma: a 2-year randomized, double-blind, placebo-controlled trial. Mayo Clin Proc 2004;79:458-66.

17. Li JTC, Ford LB, Chervinsky $P$, et al. Fluticasone propionate powder and lack of clinically significant effects on hypothalamic-pituitary-adrenal axis and bone mineral density over 2 years in adults with mild asthma. J Allergy Clin Immunol 1999;103:1062-8.

18. Maspero J, Backer V, Yao R, et al. Effects of mometasone, fluticasone, and montelukast on bone mineral density in adults with asthma. J Allergy Clin Immunol Pract 2013;1:649-55.e1.

19. Roux C, Kolta S, Desfougeres JL, et al. Long-term safety of fluticasone propionate and nedocromil sodium on bone in children with asthma. Pediatrics 2003;111(6 Pt 1):e706-13.
20. Turpeinen M, Pelkonen AS, Nikander $\mathrm{K}$, et al. Bone mineral density in children treated with daily or periodical inhaled budesonide: the Helsinki early intervention childhood asthma study. Pediatr Res 2010;68:169-73.

21. Agertoft L, Pedersen S. Bone mineral density in children with asthma receiving long-term treatment with inhaled budesonide. Am J Respir Crit Care Med 1998;157:178-83.

22. Allen HDW, Thong IG, Clifton-Bligh $P$, et al. Effects of high-dose inhaled corticosteroids on bone metabolism in prepubertal children with asthma. Pediatr Pulmonol 2000;29:188-93.

23. Bahceciler NN, Sezgin G, Nursoy MA, et al. Inhaled corticosteroids and bone density of children with asthma. J Asthma 2002;39:151-7.

24. El O, Gulbahar S, Ceylan E, et al. Bone mineral density in asthmatic patients using low dose inhaled glucocorticosteroids. J Investig Allergol Clin Immunol 2005;15:57-62.

25. Harris M, Hauser S, Nguyen TV, et al. Bone mineral density in prepubertal asthmatics receiving corticosteroid treatment. $J$ Paediatr Child Health 2001;37:67-71.

26. Johannes CB, Schneider GA, Dube TJ, et al. The risk of nonvertebral fracture related to inhaled corticosteroid exposure among adults with chronic respiratory disease. Chest 2005;127:89-97.

27. Schlienger RG, Jick SS, Meier CR. Inhaled corticosteroids and the risk of fractures in children and adolescents. Pediatrics 2004:114:469-73.

28. Sosa M, Saavedra $\mathrm{P}$, Valero $\mathrm{C}$, et al. Inhaled steroids do not decrease bone mineral density but increase risk of fractures: data from the GIUMO study group. J Clin Densitom 2006;9:154-8.

29. Van Staa TP, Bishop N, Leufkens HGM, et al. Are inhaled corticosteroids associated with an increased risk of fracture in children? Osteoporos Int 2004:15:785-91.

30. Wisniewski AF, Lewis SA, Green DJ, et al. Cross sectional investigation of the effects of inhaled corticosteroids on bone density and bone metabolism in patients with asthma. Thorax 1997;52:853-60.

31. Yanik B, Ayrim A, Ozol D, et al. Influence of obesity on bone mineral density in postmenopausal asthma patients undergoing treatment with inhaled corticosteroids. Clinics 2009;64: 313-18.

32. The Childhood Asthma Management Program Research Group. Long-term effects of budesonide or nedocromil in children with asthma. N Engl J Med 2000;343:1054-63.

33. Richy F, Bousquet J, Ehrlich GE, et al. Inhaled corticosteroids effects on bone in asthmatic and COPD patients: a quantitative systematic review. Osteoporos Int 2003;14:179-90. 